Publ. RIMS, Kyoto Univ.

13 (1977), 301-305

\title{
A Five-Square Theorem
}

\author{
By \\ Saburô UCHIYAMA*
}

T'urán Pál in memoriam

It is clear that for every even integer $2 n>0$ there is a natural number $s$ such that $2 n$ is representable in the form

$$
2 n=\sum_{i=1}^{s} x_{i}{ }^{2} \text { with the condition } \sum_{i=1}^{s} x_{i}=0,
$$

where the $x_{i}(1 \leqq i \leqq s)$ are rational integers. We denote by $s(2 n)$ for a given $2 n$ the smallest possible value of such $s$. Of course, no representations of that kind are possible for odd integers.

We have evidently $2 \leqq s(2 n) \leqq 8$ for all $2 n>0$. The purpose of this note is to prove the following

Theorem. We have

$$
s(2 n) \leqq 5 \text { for all } 2 n>0
$$

with the equality exclusively for the integers 2 of the form

$$
4^{k}(32 l+28) \quad(k \geqq 0, l \geqq 0) .
$$

The problem of determining the value of

$$
\max _{n \geqq 1} s(2 n)
$$

has been (orally) communicated to the writer by Professor S. Hitotumatu in Kyoto University, who was led to this problem in the course of his study of 'translatable complete $l$-th power configurations.' Our result gives a satisfactory solution for the problem proposed.

It should be noted, horvever, that a general problem on the solvability of the system of Diophantine equations

Communicated by S. Hitotumatu, October 25, 1976.

* Department of Mathematics, Okayama University, Okayama, 700 Japan. 


$$
n=\sum_{i=1}^{s} x_{i}^{2}, \quad m=\sum_{i=1}^{s} x_{i},
$$

has been treated by G. Pall [5], who showed in particular that if $s=4$ equations (3) are solvable in integers $x_{i}$, if and only if

$$
n \equiv m(\bmod 2) \text {, and } 4 n-m^{2}=\mathrm{a} \text { sum of three squares, }
$$

whereas if $s=5$ the conditions

$$
n \equiv m(\bmod 2), 4 n-m^{2} \geqq 0
$$

are necessary and sufficient for the solvability of (3) in integers $x_{i}$. (The case of $s=4$ is due substantially to A. L. Cauchy [2]. See also [4].) Our theorem is an immediate consequence of these results; but, this notwithstanding, we shall present here another simple and direct proof of the theorem.

An analogue to (1) for the representation of an odd integer $2 n+1>0$ will be

$$
2 n+1=\sum_{i=1}^{s} x_{i}^{2} \quad \text { with } \quad \sum_{i=1}^{s} x_{i}=1
$$

where the $x_{i}$ are again rational integers. If we denote by $s(2 n+1)$ for a given $2 n+1$ the smallest possible value of $s$ in the representation (4), then it can be shown that we have

$$
s(2 n+1) \leqq 4 \text { for all } 2 n+1>0 .
$$

This result also is a special case of Pall's [5].

1. In order to prove the theorem we require some auxiliary results which we formulate in the following lemma (cf. e.g. [1]).

Lemma. Let $m$ be a positive integer. The integer $m$ can be represented in the form

$$
m=x^{2}+y^{2}+z^{2}
$$

with some integers $x, y, z$, if and only if $m$ is not of the form

$$
4^{k}(8 l+7) \quad(k \geqq 0, l \geqq 0) ;
$$


the integer $m$ can be represented in the form

$$
m=x^{2}+y^{2}+2 z^{2}
$$

with some integers $x, y, z$, if and only if $m$ is not of the form

$$
4^{k}(16 l+14) \quad(k \geqq 0, l \geqq 0) .
$$

As a matter of fact, the first part of the lemma is a classical theorem proved by G. L. Dirichlet, and the second part is also a well-known result which, as has been noted by L. E. Dickson [3], can be derived easily from the first part.

Now, let there be given an even integer $2 n>0$. We shall first show that every number $2 n$ of the form (2) admits a representation of the type (1) with $s=5$. In fact, it will obviously suffice to prove that an even integer $2 n$ of the form $32 l+28(l \geqq 0)$ is representable in that form. Since $16 l+4$ is not of the form (6), we have in virtue of the lemma

$$
16 l+4=x^{2}+y^{2}+2 z^{2}
$$

for some integers $x, y, z$, and

$$
\begin{aligned}
2 n= & 32 l+28=2(16 l+4)+20 \\
= & (x+z+1)^{2}+(-x+z+1)^{2}+(y-z+1)^{2} \\
& +(-y-z+1)^{2}+(-4)^{2},
\end{aligned}
$$

as required.

Next, we shall show that if $2 n$ has the form (2), then it cannot be represented in the form (1) with $s \leqq 4$. Indeed, if we had

$$
2 n=\sum_{i=1}^{4} x_{i}^{2} \text { and } \sum_{i=1}^{4} x_{i}=0
$$

then we would have

$$
\begin{aligned}
2 n & =x_{1}{ }^{2}+x_{2}{ }^{2}+x_{3}{ }^{2}+\left(-x_{1}-x_{2}-x_{3}\right)^{2} \\
& =\left(x_{1}+x_{2}\right)^{2}+\left(x_{2}+x_{3}\right)^{2}+\left(x_{3}+x_{1}\right)^{2} ;
\end{aligned}
$$

but, this is impossible in riew of the lemma since $2 n$ is an integer of the form (5).

Finally, we prove that if $2 n$ is not of the form (2), then it is 
representable in the form (1) with $s \leqq 4$. We distinguish two cases according as $n$ is odd or even.

If $n$ is odd, then by the lemma there are integers $x, y, z$ such that

$$
n=x^{2}+y^{2}+2 z^{2}
$$

and so

$$
2 n=(x+z)^{2}+(-x+z)^{2}+(y-z)^{2}+(-y-z)^{2} .
$$

If $n$ is even, $(2 n) / 4$ is an integer which is not of the form (5) and, again by the lemma, we have

$$
2 n=4\left(x^{2}+y^{2}+z^{2}\right)=(2 x)^{2}+(2 y)^{2}+(2 z)^{2}
$$

for some integers $x, y, z$, whence, putting $2 x=x_{1}+x_{2}, 2 y=x_{2}+x_{3}, 2 z$ $=x_{3}+x_{1}$, we obtain

$$
\begin{aligned}
2 n & =\left(x_{1}+x_{2}\right)^{2}+\left(x_{2}+x_{3}\right)^{2}+\left(x_{3}+x_{1}\right)^{2} \\
& =x_{1}{ }^{2}+x_{2}{ }^{2}+x_{3}{ }^{2}+\left(-x_{1}-x_{2}-x_{3}\right)^{2} .
\end{aligned}
$$

This completes the proof of our theorem.

A simple consequence of the theorem is that the positive quaternary quadratic form

$$
x_{1}^{2}+x_{2}^{2}+x_{3}^{2}+x_{4}^{2}+x_{1} x_{2}+x_{1} x_{3}+x_{1} x_{4}+x_{2} x_{3}+x_{2} x_{4}+x_{3} x_{4}
$$

represents all positive integers (and 0 trivially).

2. We have proved that $s(2 n)=5$ if and only if the integer $2 n$ has the form (2). For the sake of completeness we should like to give a description of the properties that characterize those integers $2 n$ for which we have $s(2 n)=2,3$ or 4 . To this end, it will be convenient to introduce the symbol $q(m)$ for an integer $m \geqq 1$ to denote $m / e^{2}$, where $e^{2}$ is the largest square divisor of the integer $m . q(m)$ is thus squarefree for all $m$.

We have:

$s(2 n)=2$ if and only if $q(2 n)=2$;

$s(2 n)=3$ if and only if $q(2 n)$ is even, is greater than 2 , and does 
not contain any prime factor $p \equiv 5(\bmod 6)$.

$s(2 n)=4$ if and only if either $q(2 n)$ is odd and $2 n$ is not of the form (2), or $q(2 n)$ is even and is divisible by soine prime number $p \equiv 5$ $(\bmod 6)$.

Note that if $q(2 n)$ is an even integer then the integer $2 n$ is not of the form (2).

\section{References}

[1] Ankeny, N. C., Sums of three squares, Proc. Amer. Math. Soc., 8 (1957), 316-319.

[2] Cauchy, A. L., Oeuvres Complètes, (2) vol. 6, pp. 320-353, Gauthier-Villars, Paris (1887).

[3] Dickson, L. E., Integers represented by positive ternary quadratic forms, Bull. Amer. Math. Soc., 33 (1927), 63-70.

[4] Dickson, L. E., Two-fold generalizations of Cauchy's lemma, Amer. J. Math., 56 (1934), 513-528.

[5] Pall, G., Simultaneous quadratic and linear representation, Quart.J. Math. (Oxford), 2 (1931), 136-141. 
